\section{P3.21 PREVALENCE OF TRICHOMONAS VAGINALIS PREDICTED TO INCREASE SUBSTANTIALLY IN AUSTRALIA DUE TO REPLACEMENT OF PAP SMEARS WITH HPV TESTING FOR CERVICAL SCREENING: A MODELLING STUDY}

${ }^{1} \mathrm{BB}$ Hui, ${ }^{1} \mathrm{C}$ Reulin, ${ }^{1} \mathrm{RJ}$ Guy, ${ }^{1,2} \mathrm{~B}$ Donovan, ${ }^{3} \mathrm{JS}$ Hocking, ${ }^{1} \mathrm{MG}$ Law, ${ }^{1} \mathrm{DG}$ Regan. ${ }^{1}$ The Kirby Institute, UNSW Australia, Sydney, New South Wales, Australia; ${ }^{2}$ Sydney Sexual Health Centre, Sydney Hospital, Sydney, New South Wales, Australia; ${ }^{3}$ Melbourne School of Population and Global Health, University of Melbourne, Melbourne, Victoria, Australia

\subsection{6/sextrans-2017-053264.258}

Introduction The prevalence of trichomoniasis is very low $(\sim 0.4 \%)$ in the general Australian population. This is attributed in part to the opportunistic detection of Trichomonas vaginalis (TV) in PAP smears of women participating in the cervical screening program. From 2017 the cervical screening program will transition from cytology to human papillomavirus (HPV) testing as the primary triage. We hypothesise that as a result of this transition, and with an increasing proportion of the population vaccinated against HPV, fewer cases of TV will be detected and the prevalence will consequently increase.

Methods A mathematical model was developed to describe the transmission of TV in the general population. We assume that following the transition from cytology to HPV testing; individuals with asymptomatic TV who test negative for high-risk (HR) HPV will remain undiagnosed and untreated. We investigate the change in TV prevalence over time as the proportion of the population vaccinated against HR HPV increases. We assume ongoing coverage of $80 \%$ in the HPV vaccination program.

Results Our modelling predicts that if the prevalence of HR HPV does not change then 10 years after the transition from PAP to HPV testing, TV prevalence will have increased from $0.4 \%$ to $1.65 \%$, and after 20 years prevalence will have reached $3.83 \%$. If we assume that HR HPV prevalence continues to decrease at the current rate, TV prevalence will reach $1.68 \%$ after 10 years and $4.06 \%$ after 20 years.

Conclusion Our results suggest that TV prevalence will increase in Australia over time due to changes in the cervical screening program. The extent of this increase will depend on the impact of HPV vaccination on the prevalence of HR HPV. Consideration should be given to enhancing TV surveillance to monitor for increasing incidence. Adding a TV test to the HPV test that will be used in cervical screening and or including TV in sexual health checks for asymptomatic individuals (contingent on an observed increase in incidence) should also be considered.

\section{P3.22 PREVALENCE AND DETERMINANTS OF TOBACCO USE AMONG PATIENTS LIVING WITH HIV (PLHIV) IN KINSHASA}

B Bepouka, H Situakibanza, Kiazayawoko Florian, M Mbula. University of Kinshasa, Kinshasa - Rep.Dem.do Congo

\subsection{6/sextrans-2017-053264.259}

Introduction Worldwide the prevalence of smoking among people living with human immunodeficiency virus (HIV) is elevated compared to the general population. Tobacco use remains one of the major cardiovascular risk factors and its use in antiretroviral (ARV) treated human immunodeficiency virus infected people may lead to activation of immune cells and rendering them more susceptible to HIV. We determined the prevalence of and factors associated with tobacco use in an antiretroviral treated HIV infected congolease people.

Methods The study was a cross-sectional, conducted in Kinshasa, Democratic republic of Congo(DRC). We randomly selected patients and 9 support structures for PLHIV, from May 2015 to August 2015. Socio-demographic, tobacco and alcohol use data were collected using World Health Organisation stepwise approach to surveillance (STEPS) questionnaire. The Chi-square test was used to compare categorical variables between tobacco users and non tobacco users. The multiple logistic regression analysis was used to determine the predictors of tobacco use status.

Results On a global active file of 5724 PLHIV, 400 were included.Of 400 ARV treated HIV infected participants, 309 (77.3\%) were females and $91(22.7 \%)$ were males. The mean age of participants was 43 years. About $32(8 \%)$ of participants were tobacco users. A higher proportion of females than males $(59.4 \%$ versus $40.6 \%, p=0.521)$ used tobacco. Food insecurity (AHR: 3.349; 95\% CI: 1.378-8.142; $\mathrm{p}=0.008$ ) and alcohol consumption (AHR 3,826; 95\% CI:1,583-9,249; $\mathrm{p}=0,003)$ were significant independent predictors of tobacco use.

Conclusion Tobacco use among ARV treated HIV infected people was common. Food insecurity and alcohol consumption were the risk factors for tobacco use. There is need to scale up the awareness on how tobacco use, apart from being a risk factor for cardiovascular diseases, interferes with viral suppression despite treatment with antiretroviral drugs.

\section{P3.23 DETERMINANTS OF NON-ADHERENCE TO ANTIRETROVIRAL THERAPY IN ADULTS IN THE CITY OF KINSHASA}

${ }^{1} \mathrm{~B}$ Bepouka, ${ }^{1} \mathrm{H}$ Situakibanza, ${ }^{1} \mathrm{~F}$ Kiazayawoko, ${ }^{2} \mathrm{~N}$ Aliocha. ${ }^{1}$ University of Kinshasa, Kinshasa - Rep.Dem.do Congo; ${ }^{2}$ Cité Des Aveugles, Kinshasa - Rep.Dem.do Congo

\subsection{6/sextrans-2017-053264.260}

Introduction Adherence of $95 \%$ or more to antiretroviral therapy is generally considered necessary for optimal virologic suppression in patients living with HIV/AIDS (PLWHA). In the African context of access followed by low viral load, understand the determinants of poor adherence is essential to improve compliance, optimise virologic suppression and reduce morbidity and mortality.

Method A transversal analytical study referred to was conducted on patients aged at least 18 years on antiretroviral treatment for at least three months. We randomly selected patients and 9 support structures for PLWHA from 63 following more than 100 patients in Kinshasa, from May 2015 to August 2015. The Case Adherence Index (subjective method) and renewal of order (method objective) were used as indicators of compliance. Khi-2 and Student tests were used for comparisons. The determinants of nonadherence were sought in multivariate logistic regression analysis.

Results On a global active file of 5724 patients, 400 were included. The median age was 43 years and the sex ratio in favour of the three women. The overall incidence of non compliance was $25 \%$. The prevalence of non-objective compliance was higher than that of non-subjective compliance $(29 \%$ vs $21 \%, p=0.01)$. In multivariate analysis, the payment of the consultation [adjusted OR $1.70 \quad 95 \%$ CI (1.020 to 2.813), 
$\mathrm{p}=0.042$ ], side effects [adjusted OR 2.23 95\% CI $(1.32$ to 3.74), $\mathrm{p}=0.002$ ] and lack of perception that the missed dose can aggravate the disease [adjusted OR 4.15, 95\% CI (1.03 to 16.67), $\mathrm{p}=0.045$ ] have emerged as determinants of the overall non-adherence.

Conclusion The results are in accordance with what has already been shown. The discrepancy observed between the two methods highlights the importance of access to biological methods. The identified risk factors will through regular evaluation, better identify PLWHA at greater risk of non-compliance and to offer enhanced compliance support.

\section{P3.24 LYMPHOGRANULOMA VENEREUM PROCTITIS ARE STILL INCREASING IN FRANCE}

'Bertille De Barbeyrac, ${ }^{2}$ Arabella Touati, ${ }^{2}$ Cécile Laurier-Nadalié, ${ }^{3}$ Chloé Le Roy, ${ }^{4}$ Nadège Hénin, ${ }^{4}$ Olivia Peuchant, ${ }^{5}$ Ndeindo Ndeikoundam, ${ }^{4}$ Cécile Bébéar, ${ }^{6}$ Guy La Ruche. 'University, USC Ea3671 Mycoplasmal and Chlamydial Infections in Humans, and National Reference Cente, Bordeaux, France; '2University, USC Ea3671 Mycoplasmal and Chlamydial Infections in Humans, and National Reference Cente, Bordeaux, France; ${ }^{3}$ University, USC Ea3671 Mycoplasmal and Chlamydial Infections in Humans, and National Reference Cente, Bordeaux, France; ${ }^{4}$ University, USC Ea3671 Mycoplasmal and Chlamydial Infections in Humans, and National Reference Cente, Bordeaux, France; ${ }^{5}$ French Institute for Public Health Surveillance, Saint Maurice, France; ${ }^{6}$ French Institute for Public Health Surveillance, Bordeaux, France

\subsection{6/sextrans-2017-053264.261}

Introduction Following the emergence of lymphogranuloma venereum (LGV) proctitis in the Netherlands in 2003, a voluntary surveillance system for LGV has been established in France. Based on the data of the National Reference Centre (NRC) for chlamydiae, Bordeaux, France, this study aimed to describe the epidemiology of LGV and non-LGV ano-rectal infection in France and to examine the characteristics of affected populations.

Methods The French surveillance network includes clinicians, biologists and NRC. Biologists sent rectal CT-positive samples to the NRC where the strains were typed by using two realtime PCR targeting $p m p \mathrm{H}$ gene specific of $\mathrm{L}$ and $\mathrm{L} 2 \mathrm{~b}$ strains. Biologists and clinicians performed a document on patient's clinical, biological and behavioural data. Clinical, biological and sexual risk behavioural variables were compared in men with LGV and with non-LGV cases according the HIV status using appropriate statistical tests over the period 2010-2015.

Results A total of 2627 LGV cases and 2633 non LGV cases were recorded from 2004 to 2015. In the period 2010 to 2015, there were 1747 LGV episodes in 1570 patients, most of whom were known to be HIV-positive (841/1105, 76.1\%) and 2242 non LGV episodes in 2051 patients, most of whom were known to be HIV-negative (1186/1665, 71.2\%). The number of LGV diagnoses was multiplied by 2.4 between 2012 and 2015 and the number of recurrence reached 10\% in 2015. LGV continues to affect a core group: HIV-infected MSM who engage in high-risk sexual practices. They were older and more often infected with syphilis than men with non-LGV cases, were usually symptomatic and mainly lived in Paris. Those who acquired LGV reinfection had concurrent hepatitis $\mathrm{C}$ and syphilis more often than those with a single episode.

Conclusion A steady annual increase in the number of LGV cases and in the number of LGV recurrences was observed since 2012, demonstrating that the LGV epidemic is not under control and requires providing better information about the disease to affected patients and physicians of all specialties who take care them

\section{P3.25 QUANTIFICATION OF THE RISK OF PELVIC INFLAMMATORY DISEASE FOLLOWING A CHLAMYDIA TRACHOMATIS TEST BY DIAGNOSTIC TEST TYPE}

${ }^{1}$ Bethan Davies, ${ }^{2}$ Katy Me Turner, ${ }^{3}$ Berit Andersen, ${ }^{4}$ Henrik Westh, ${ }^{1}$ Helen Ward. ${ }^{1} /$ mperial College London, London - Great Britain; ${ }^{2}$ University of Bristol, Bristol - Great Britain; ${ }^{3}$ Department of Public Health Programmes, Randers - Denmark; ${ }^{4}$ Hvidovre University Hospital, Copenhagen - Denmark

\subsection{6/sextrans-2017-053264.262}

Introduction Nucleic Acid Amplification Tests (NAATs) are the recommended test type for diagnosing Chlamydia trachomatis (chlamydia). However poorer performing methods remain in use. We compared the risk of pelvic inflammatory disease (PID) in women tested for chlamydia by diagnostic test type.

Methods We used a sub-set of the Danish Chlamydia study that included all female residents of Denmark who were tested for chlamydia (1998-2001) when aged 15-34 years. Chlamydia tests performed on urinary or genital samples with a definitive positive or negative result were categorised as non-NAAT (ELISA; IF; “antigen") or NAAT (PCR; SDA; TMA; LCR; DNA/RNA) and limited to each woman's first test. Test records were linked to hospital presentations for PID within 12 months. Women with previous PID or PID diagnosed on the same date as the test were excluded. We used logistic regression to compare the risk of PID by test type adjusted for age, test year and test result.

Results Of the 272105 women in the study, $44.78 \%$ were tested using NAAT, $6.38 \%$ tested positive for chlamydia and $0.64 \%$ were diagnosed with PID within 12 months. Overall, the adjusted risk of PID within 12 months of a chlamydia test was higher following a positive test (AOR 1.40 (95\% CI 1.18$1.67)$ and in older women (25-34 years $1.36(1.23-1.49)$ ) and lower in women tested using a NAAT $(0.87(0.78-0.96))$ and in the more recent time interval (2000/2001 $0.89(0.80$ 0.99)). In women with a positive test, and presumably treated infection, the risk of PID did not differ by test type (1.25 (0.87-1.79)). In women with a negative test, the risk of PID was lower following a NAAT $(0.84(0.75-0.93))$.

Conclusion Women with a negative result from a non-NAAT chlamydia test have a $16 \%$ higher risk of PID by 12 months compared to women with a negative result from a NAAT. This is presumably due to the increased proportion of false negative tests with the less sensitive non-NAATs. This study quantifies the health impact of using poorer performing chlamydia diagnostic tests and provides further evidence for phasing them out.

\section{P3.26 THE EFFICACY OF AZITHROMYCIN AND DOXYCYCLINE TREATMENT FOR RECTAL CHLAMYDIAL INFECTION: A RETROSPECTIVE COHORT STUDY IN SOUTH AUSTRALIA}

$\mathrm{B} \mathrm{Li}{ }^{1,2},{ }^{3} \mathrm{JS}$ Hocking, P Bi ${ }^{1}, \mathrm{C}$ Bell ${ }^{2}$, A Ward ${ }^{2}, \mathrm{CK}$ Fairley ${ }^{4,5} .{ }^{1}$ The University of Adelaide, Australia; ${ }^{2}$ Royal Adelaide Hospital, Australia; ${ }^{3}$ University of Melbourne, Australia; ${ }^{4}$ Monash University, Australia; ${ }^{5}$ Melbourne Sexual Health Centre, Australia

\subsection{6/sextrans-2017-053264.263}

Introduction British Association for Sexual Health and HIV (BASHH) and Australian guidelines recommend either 